\title{
Computer Analysis of Lighting in Realist Master Art: \\ Current Methods and Future Challenges
}

\author{
David G. Stork \\ Ricoh Innovations, 2882 Sand Hill Road, Menlo Park CA 94025, USA \\ and Department of Statistics, Stanford University, Stanford CA 94305, USA \\ artanalyst@gmail.com
}

\begin{abstract}
We review several computer based techniques for analyzing the lighting in images that have proven valuable when addressing questions in the history and interpretation of realist art. These techniques fall into two general classes: model independent (where one makes no assumption about the three-dimensional shape of the rendered objects) and model dependent (where one does make some assumptions about their three-dimensional shape). An additiona, statistical algorithm integrates the estimates of lighting position or direction produced by different such techniques. We conclude by discussing several outstanding problems and future directions in the analysis of lighting in realist art.
\end{abstract}

Keywords: computer vision, computer image analysis of art, occluding contour algorithm, cast shadow analysis, computer graphics constructions, tableau virtuel.

\section{Introduction}

In the past several years, algorithms from computer vision and image analysis have been extended and applied to an ever widening range of questions in the history of art. These algorithms have been applied to challenges in low-level imaging (to enhance the visibility of hidden or degraded passages, to predict the effects of conservatorial treatment of artworks, to identify pigments by spectral signature, to rejuvenate appearance by digitally reversing the fading of pigments, ...), stroke and mark analysis (to infer the marking tool, to date intaglio prints as the master wears, to identify the number of artists's "hands" in a given work, ...), perspective analysis (to reveal perspectival inaccuracies to test for artists' use of optical aids, ...), contour shape analysis (to test fidelity of art copies, ...), composition analysis (to infer compositional principles and detect forgeries, ...), computer graphics reconstructions (to infer sight lines, infer artists' studio practice, ...).

Another, important, general class of problems involve estimating the position or direction of illumination based on the image information in a painting, the class we consider here. Finding the direction or position of an illuminant can 
help answer a number of problems in the history of art. For example, Johnson, Stork, Biswas and Furuichi estimated the direction of illumination in Jan Vermeer's Girl with a pearl earring using six semi-independent methods. 1 The excellent agreement among these estimates very strongly implies that Vermeer had an actual figure in his studio rather than worked from memory or created and ideal "fictive" figure. Stork and colleagues used several methods to estimate the location of the illuminant in Georges de la Tour's Christ in the carpenter's studio to test the claim that this painting was executed using optically projected images. 203|4|5] In those works, the illuminant could be assumed a point source, or fairly distant from the objects in question. Stork and Johnson extended the algorithms to the case of diffuse illumination, and tested paintings by the contemporary realist Garth Herrick, specifically Apotheon and Human on my faithless arm to reveal the painterly analogy of "compositing," here working from two photographic referents having different lighting conditions. [6]

Here we summarize some of the techniques and how they have addressed problems in the history of art.

The two general classes of methods are: model independent methods (where one makes no assumptions about the three-dimensional shape of the depicted objects) and model dependent methods (where one does make assumptions about the three-dimensional shape). We review these in Sects. 2] and 3, respectively, then turn in Sect. 4 to statistical and computational methods for integrating estimates from disparate information sources. We list some outstanding problems and future directions in Sect. 5 .

\section{Model Independent Methods}

In model independent algorithms, one does not need to know or assume the threedimensional shape of the depicted objects. All the visual information necessary for the algorithm can be extracted directly from the two-dimensional image itself. One may need to make assumptions or have other knowledge, for instance that the objects are of uniform albedo or reflectivity - but not assumptions about the shape.

\subsection{Cast Shadow Analysis}

The simplest technique for determining the direction of point-source illumination in the plane perpendicular to the light of sight is cast-shadow analysis. One merely identifies a point on an occluder, such as a tip of a nose or a finger, and its corresponding point on the cast shadow, draw a line linking them. This line, extended, should go through the two-dimensional location of the point-source illuminant. [7/2]

\subsection{Occluding Contour Analysis}

The occluding contour algorithm takes as input the measured lightness along the outer boundary or occluding contour of a three-dimensional object, and returns 
the direction to the illumination that best explains (in a least-square sense) this pattern. The algorithm can be applied only to contours of Lambertian surfaces (diffusely reflecting like cloth or skin, not shiny like glass or metal), and where the surface is of uniform albedo or reflectivity. Moreover, the contours must be convex and fairly far from other objects to avoid scattered light. 8

Stork and Johnson pioneered the use of this algorithm in the study of art, showing that the illumination in the tableau in Georges de la Tour's Christ in the carpenter's studio came from the depicted candle, not (as had been claimed) from outside the picture, or from "in place of the other figure." 79 Johnson et al. applied the algorithm to Jan Vermeer's Girl with a pearl earring and found the direction to the illumination determined by this algorithm matched quite closely the directions estimated by several other methods. [1] This was an objective testament to both the power of these lighting estimation methods, but also to Vermeer's mastery in rendering the effects of light.

While the above work assumed, with great justification, that the light source was distance or a point source, most paintings depict scenes in which the tableau is illuminated by complex illumination. As such, Stork and Johnson extended the algorithms to this more complicated case, where the incident light could be represented as a series of five spherical harmonics. They used this representation to test paintings by Garth Herrick for consistency in lighting on different figures. The algorithm could indeed reveal that different figures had been rendered under different illumination conditions. 6] This technique might be applicable to other realist works, for instance Renaissance portraits containing multiple figures, where art historians are unsure how many artists - "hands" - executed the work.

\section{Model Dependent Methods}

As mentioned above, in model dependent algorithms, one knows or assumes the three-dimensional shape of the depicted objects. Sometimes the models are simple - as in a sphere or eyeball. Other times they can be quite complex - as in a full three-dimensional computer graphics model.

\subsection{Specular Reflection}

Specular reflections, such as the highlights on eyes, can be used to infer the direction of illumination. Johnson et al. used the highlights on the eyes in Jan Vermeer's Girl with a pearl earring to infer the direction of illumination. 1] They found this direction matched quite well the directions estimated from several other model and model independent methods.

\subsection{Diffuse Reflection from Plane Surfaces}

Yet another method for inferring the position of the illuminant is based on the pattern of lightness on planar surfaces, for instance a floor or wall. Stork and Kale derived the equations for the appearance model of a planar Lambertian surface 
(such as the floor inGeorges de la Tour's Christ in the carpenter's studio and used it to estimate the position of a point source consistent with the pattern of lightness found on the floor. 510 Although the results here were not as definitive as other methods in this painting, the results supported the claim that the light was in place of the candle rather than in place of the other figures.

\subsection{Computer Graphics Rendering}

Although computer graphics reconstructions have been used for exploring geometry, perspective, sight lines, and so on, these techniques have also been used to explore studio lighting conditions. Johnson et al. built a computer graphics model of Girl with a pearl earring, 1] Stork and Furuichi built a model of Christ in the carpenter's studio, Stork explored a model of Caravaggio's Calling of St. Matthew, [1] and finally Stork and Furuichi built a model of Diego Velàzquez's Las meninas. 12] In all these cases, the experimenters could adjust the location of virtual illuminants in the model (or tableau virtuel) until the rendered image matched the corresponding painting as closely as possible. In this way, these scholars could answer questions about studio practice, for instance whether some artists secretly traced optically projected images.

\subsection{Shape from Shading}

Johnson et al. exploited shape-from-shading methods to infer the direction of illumination in Vermeer's Girl with a pearl earring. 1] Because they did not have an accurate three-dimensional model of the girl's face, they adjusted a generic three-dimensional face model to match that of the girl. They temporarily assumed this was the actual model, and then inferred the direction of illumination that, for this model, best fit the measured lightness in the painting. This gave an initial estimate to the direction of illumination.

This iterative scheme is an example of the expectation-maximization (EM) algorithm, or more specifically the generalized expectation maximization (GEM) algorithm. 13. The direction they found closely matched that found using other lighting estimation methods, a testament to Vermeer's mastery in rendering the effects of light, and further corroboration that there was almost surely an actual model in his studio.

\section{Integration of Lighting Estimates}

Given several estimates of the direction or location of an illuminant, the problem arises how to integrate them to a single estimate. The principled, statistical method is Bayesian integration, [13] where here (under very reasonable assumptions) the integration is computed by:

$$
p(\mathbf{x} \mid \Theta) \propto \prod_{i}^{k} P_{i} p\left(\mathbf{x} \mid \theta_{i}\right) .
$$


That is, the probability density that the point illuminant is in spatial location $\mathbf{x}$ given all the estimates, $\boldsymbol{\Theta}$, is proportional to the product of the densities from each individual estimates, $p\left(\mathbf{x} \mid \theta_{i}\right)$, multiplied by their prior probabilities or confidences, $P_{i}$. This method has been used to integrate estimates in de la Tour's Christ in the carpenter's studio. 7 .

\section{Conclusions and Future Directions}

There are a number of outstanding computational problems that must be solved so as to extend the power of these methods and extend their applicability to a wider range of artworks and problems in the history of art. The first is to get a better estimate of the errors of each of the techniques when applied to a given work. Some of the methods, such as the occluding contour algorithm and cast shadow analysis, yield estimates of one spatial angle, perpendicular to the viewer's (and artist's) line of sight, while others, such as shape-from-shading and computer graphics modelling, yield estimates in two spatial angles. We need rigorous and principled methods for integrating such estimates, particularly with their errors. An intriguing problem is to generalize the occluding contour based methods in diffuse lighting environments to the case of multiple colored illuminants, for instance an orange candle and blue sky flowing through a window.

This work in lighting in art is part of a broader research effort that is leading to new techniques and shedding new light on art and art praxis. 1415]

\section{Acknowledgments}

The author gratefully acknowledges insights provided by numerous scientists, conservators, and historians of optics and art, as well as by anonymous reviewers, and would also like to thank the Getty Research Institute, Santa Monica CA, for extended reader's privileges in its superb research library.

\section{References}

1. Johnson, M.K., Stork, D.G., Biswas, S., Furuichi, Y.: Inferring illumination direction estimated from disparate sources in paintings: An investigation into Jan Vermeer's Girl with a pearl earring. In: Stork, D.G., Coddington, J. (eds.) Computer image analysis in the study of art, vol. 6810. SPIE/IS\&T, Bellingham, 68100I-1-12 (2008)

2. Stork, D.G.: Did Georges de la Tour use optical projections while painting Christ in the carpenter's studio? In: Said, A., Apolstolopoulos, J.G. (eds.) SPIE Electronic Imaging: Image and video communications and processing, vol. 5685, pp. 214-219. SPIE, Bellingham (2005)

3. Stork, D.G.: Locating illumination sources from lighting on planar surfaces in paintings: An application to Georges de la Tour and Caravaggio. In: Optical Society of American Annual Meeting, Rochester, NY, OSA (2008) (abstract) 
4. Stork, D.G., Furuichi, Y.: Image analysis of paintings by computer graphics synthesis: An investigation of the illumination in Georges de la Tour's Christ in the carpenter's studio. In: Stork, D.G., Coddington, J. (eds.) Computer image analysis in the study of art, vol. 6810. SPIE/IS\&T, Bellingham, 68100J-1-12 (2008)

5. Stork, D.G.: Locating illumination sources from lighting on planar surfaces in paintings: An application to Georges de la Tour and Caravaggio. In: Optical Society of American Annual Meeting, Rochester, NY, Optical Society of America (2008)

6. Stork, D.G., Johnson, M.K.: Lighting analysis of diffusely illuminated tabeaus in realist paintings: An application to detecting 'compositing' in the portraits of Garth Herrick. In: Delp III, E.J., Dittmann, J., Memon, N.D., Wong, P.W. (eds.) Electronic Imaging: Media forensics and security XI, vol. 7254. SPIE/IS\&T, Bellingham, 72540L1-8 (2009)

7. Stork, D.G., Johnson, M.K.: Estimating the location of illuminants in realist master paintings: Computer image analysis addresses a debate in art history of the Baroque. In: Proceedings of the 18th International Conference on Pattern Recognition, Hong Kong, vol. I, pp. 255-258. IEEE Press, Los Alamitos (2006)

8. Nillius, P., Eklundh, J.-O.: Automatic estimation of the projected light source direction. In: IEEE Conference on Computer Vision and Pattern Recognition (CVPR 2001), vol. 1, pp. 1076-1083 (2001)

9. Stork, D.G., Johnson, M.K.: Computer vision, image analysis and master art, Part II. IEEE Multimedia 14(3), 12-17 (2006)

10. Kale, D., Stork, D.G.: Estimating the position of illuminants in paintings under weak model assumptions: An application to the works of two Baroque masters. In: Rogowitz, B.E., Pappas, T.N. (eds.) Electronic Imaging: Human vision and electronic imaging XIV, vol. 7240. SPIE/IS\&T, Bellingham, 72401M1-12 (2009)

11. Stork, D.G.: New insights into Caravaggio's studio methods: Revelations from computer vision and computer graphics modeling. Renaissance Society of American Annual Meeting Los Angeles, CA, p. 102 (2009) (abstract)

12. Stork, D.G., Furuichi, Y.: Computer graphics synthesis for interring artist studio practice: An application to Diego Velázquez's Las meninas. In: McDowall, I.E., Dolinsky, M. (eds.) Electronic imaging: The engineering reality of virtual reality, vol. 7238. SPIE/IS\&T, Bellingham, 7238061-9 (2009)

13. Duda, R.O., Hart, P.E., Stork, D.G.: Pattern classification, 2nd edn. John Wiley and Sons, New York (2001)

14. Stork, D.G., Coddington, J. (eds.): Computer image analysis in the study of art, vol. 6810. SPIE/IS\&T, Bellingham (2008)

15. Stork, D.G., Coddington, J., Bentkowska-Kafel, A. (eds.): Computer vision and image analysis of art. SPIE/IS\&T, Bellingham (forthcoming, 2010) 